\title{
Presión con manómetro del neumotaponamiento del tubo endotraqueal en pacientes adultos en el servicio de urgencias
}

\author{
Claudia J. Merlos-Villegas ${ }^{1}$, Ricardo Bañuelos-Huerta ${ }^{1}$, Jazmín García-Machorro ${ }^{2}$ y \\ Ma. de la Luz León-Vázquez ${ }^{3 *}$
}

${ }^{1}$ Departamento de Urgencias, Hospital General de Zona N. ${ }^{\circ}$, Instituto Mexicano del Seguro Social, Tlaxcala; ${ }^{2}$ Departamento de Revisores externos, Escuela Superior de Medicina, Instituto Politécnico Nacional, Ciudad de México; ${ }^{3}$ Departamento de Coordinación de investigación, Instituto Mexicano del Seguro Social Delegación Tlaxcala, Tlaxcala. México

\begin{abstract}
Resumen
Introducción: La intubación traqueal, además de formar parte de la atención a pacientes críticos, puede conllevar diferentes complicaciones. Una presión inadecuada dentro del manguito del tubo endotraqueal es el principal factor de riesgo. Objetivo: Evaluar la presión del neumotaponamiento del tubo en los pacientes adultos en el servicio de urgencias. Métodos: Diseño observacional, analítico. Se analizaron 60 procedimientos de intubación endotraqueal por personal médico de urgencias. La presión del neumotaponamiento fue calculada de forma empírica, posteriormente registrada con un manómetro en $\mathrm{cmH}_{2} \mathrm{O}$. Se compararon las diferentes categorías con la presión de insuflado. Se utilizó análisis descriptivo, chi cuadrada de Pearson y ANOVA de un factor. Se consideró significativo un grado de significación (p) menor a 0.05 . Resultados: Se incluyeron 60 pacientes. El $77 \%$ fueron neumotaponamientos suprainsuflados y el $23 \%$ normoinsuflados. El $64 \%$ de las intubaciones las realizaron residentes de $3 .{ }^{\text {er }}$ año de urgencias. El volumen administrado al neumotaponamiento fue entre 4 y $10 \mathrm{ml}$. La presión de insuflado fue de 22 a $45 \mathrm{cmH}_{2} \mathrm{O}$, sin diferencia significativa entre el personal que manipuló el globo. Conclusiones: La presión del neumotaponamiento del tubo endotraqueal insuflado de manera empírica en los pacientes adultos en el servicio de urgencias es mayor al límite normal.
\end{abstract}

Palabras clave: Anestesia. Endotraqueal. Terapia. Emergencia.

\section{Pressure with manometer of endotracheal tube pneumobulation in adult patients} in the emergency department

\begin{abstract}
Introduction: Tracheal intubation, in addition to being part of the care of critically ill patients, can lead to different complications. Inadequate pressure within the endotracheal tube cuff is the main risk factor. Objective: The objective of this study was assess the pressure of the pneumoblogging of the tube in adult patients in the emergency department. Methods: Observational, analytical design. Sixty endo-tracheal intubation procedures were analyzed by emergency medical personnel. Pneumoblog pressure was calculated empirically, subsequently recorded with a manometer in $\mathrm{cmH}_{2} \mathrm{O}$. The different categories were compared with the insufflation pressure. Descriptive analysis, Pearson's chi square and one-way ANOVA were used. A p value less than 0.05 was considered significant. Results: 60 patients were included. $77 \%$ were suprainflated pneumo-
\end{abstract}

\section{Correspondencia:}

*María de la Luz León-Vázquez

E-mail: clarissa023@gmail.com
Disponible en internet: 10-11-2021

Fecha de recepción: 24-03-2021

Fecha de aceptación: 26-08-2021 DOI: 10.24875/REIE.21000024
Rev Educ Investig Emer. 2022;4(1):5-10 www.medicinadeemergencias.com

2604-6520 @ 2021 Sociedad Mexicana de Medicina de Emergencias, AC. Publicado por Permanyer México SA de CV. Este es un artículo open access bajo la licencia CC BY-NC-ND (http://creativecommons.org/licenses/by-nc-nd/4.0/). 
tamples and $23 \%$ were normal inflated. $64 \%$ of intubations were performed by residents of the $3^{\text {rd }}$ year of the emergency room. The volume administered to the pneumobag was between 4 and $10 \mathrm{ml}$. The insufflation pressure ranged from 22 to $45 \mathrm{cmH}_{2} \mathrm{O}$, with no significant difference between the personnel who handled the balloon. Conclusions: The pressure of the empirically inflated endotracheal tube pneumotracheal tube in adult patients in the emergency department is greater than the normal limit

Keywords: Anaesthetic. Endotracheal. Terapia. Emergency.

\section{Introducción}

La intubación traqueal, además de formar parte de la atención a pacientes críticos y anestesiados, puede conllevar diferentes complicaciones, el tubo endotraqueal (TET) es uno de los principales factores de riesgo'; una insuflación inadecuada, tanto excesiva como deficiente, está relacionada con estos, por lo que es necesario mantener una presión adecuada del manguito para reducir el riesgo de inhalación de secreciones ${ }^{2}$.

A pesar de las modificaciones que se han hecho a Ios TET para evitar la aspiración y tener una ventilación con presión positiva; continúa siendo insuficiente ${ }^{2}$.

La estimación por palpación es la técnica más rápida y utilizada en los diferentes servicios, sobre todo en salas de emergencias, siendo ocupada hasta en más de un $80 \%$ de las veces en los actos de intubación; sin embargo este método para estimar la presión dentro del manguito es inadecuado, presentando en la mayoría de los grupos estudiados una sobreinsuflación, independiente del sexo 0 talla del paciente e incluso de la experiencia del personal, estableciendo la necesidad de mediciones objetivas y abandono de técnicas subjetivas $^{1,3}$.

Si no hay una detección de la presión del neumotaponamiento de forma temprana, cuando esta se encuentra por encima de las cifras consideradas como normales puede haber una lesión importante en la mucosa de la tráquea periférica al neumotaponamiento, dichas lesiones pueden presentarse desde los primeros minutos de la insuflación ${ }^{4}$.

Desde hace varias décadas se dispone de manómetros para evaluar y mantener las presiones recomendadas del globo endotraqueal ${ }^{5}$, dichos dispositivos no son de uso común, casi todas las investigaciones relacionadas son realizadas en servicios de anestesiología.

No hay estudios acerca del uso del manómetro para determinar la presión de insuflado del neumotaponamiento en el área de urgencias. Por lo que el objetivo del estudio fue determinar la presión del neumotaponamiento del TET insuflado de manera empírica en pacientes adultos a fin de evidenciar que la palpación no es un método confiable para determinar la insuflación adecuada del neumotaponamiento.

\section{Pacientes y método}

Se trata de un estudio observacional, analítico, prospectivo. Realizado posterior a la aprobación por los comités de ética en investigación y comité local de investigación 2902 con registro R-2020-2902-024. La población incluida fue personal médico (base y residentes de especialidad en urgencias médico-quirúrgicas), quienes realizaron intubación en el servicio de urgencias, previa capacitación sobre el uso del manómetro. El muestreo fue no probabilístico por casos consecutivos.

\section{Procedimiento}

Una vez realizado el procedimiento de intubación con secuencia rápida, se verificó el posicionamiento adecuado del TET en vía aérea y se realizó la fijación de este por parte del servicio de enfermería; una vez colocado y fijado, los pacientes fueron conectados al ventilador mecánico con los parámetros previamente calculados e individualizados. Posterior a estabilización del paciente, se realizó por parte del médico de urgencias la medición de la presión del neumotaponamiento con un manómetro aneroide para control de presión Universal VBM, escala con diámetro de $68 \mathrm{~mm}$, escala para TET (20-30 $\left.\mathrm{cmH}_{2} \mathrm{O}\right)$ en los primeros $30 \mathrm{mi}$ nutos posteriores a la intubación, registrando la presión exacta de la medición en $\mathrm{cmH}_{2} \mathrm{O}$, considerándose adecuada entre 20 y $30 \mathrm{cmH}_{2} \mathrm{O}^{6}$ y su conversión a $\mathrm{mmHg}$ (15-22 mmHg). Se registró además el volumen en $\mathrm{ml}$ administrado para neumotaponamiento y el personal que realizó el procedimiento (médico con especialidad en urgencias, médico con otra cualquier otra especialidad o médicos residentes de urgencias médico-quirúrgicas de los diferentes años).

Con base en la presión registrada se realizó una clasificación distinguiendo los que fueron infrainsuflados, adecuadamente insuflados o suprainsuflados; una vez determinado, el personal a cargo realizó la 
corrección de la presión en el TET con el manómetro en todos los casos en los que se hubiera detectado errores de insuflación.

\section{Análisis estadístico}

Para el procesamiento de los datos se utilizó el programa estadístico SPSS versión 24. Se realizó un análisis descriptivo con porcentajes y frecuencias para las variables sexo, categoría del personal e insuflación; para el volumen y presión de insuflado se utilizó media y desviación estándar. Para comparar la presión del neumotaponamiento con el personal que realizó el procedimiento se utilizó prueba ANOVA de un factor y chi cuadrada de Pearson.

\section{Resultados}

Se analizaron un total de 60 procedimientos en el área de urgencias. El $64 \%$ de las intubaciones e insuflaciones del neumotaponamiento fue realizado por médicos residentes de tercer año de la especialidad en urgencias (Tabla 1).

El volumen administrado al neumotaponamiento fue de 4 a $10 \mathrm{ml}$ (media: 6; desviación estándar [DE]: $1.5 \mathrm{ml}$; intervalo de confianza del 95\% [IC 95\%]: 5.9$6.6)$; el $37 \%$ recibió $5 \mathrm{ml}$ (Tabla 2). El volumen en $\mathrm{ml}$ del neumotaponamiento normoinsuflado fue de $5 \pm$ $0.7 \mathrm{ml}$ (IC 95\%: 4.5-5.3), mientras que en el suprainsuflado de $6.6 \pm 1.4 \mathrm{ml}$ (IC 95\%: 6.3-7).

La presión de insuflado fue de 22 a $45 \mathrm{cmH}_{2} \mathrm{O}$, con una media de $33 \pm 4 \mathrm{cmH}_{2} 0$, correspondiendo a $24 \pm$ $3 \mathrm{mmHg}$. El volumen en mililitros de aire introducido para la insuflación del neumotaponamiento tiene una correlación directa con la presión del insuflado (coeficiente de correlación: 0.904).

En ninguno de los pacientes intubados se detectó un insuflado por debajo del límite normal, 46 neumotaponamientos estuvieron suprainsuflados (77\%) y 14 normoinsuflados (23\%).

No se encontró diferencia significativa entre el personal que manipuló el globo y el grupo normoinsuflado vs. el suprainsuflado ( $p=0.9$ ) (Fig. 1).

Al comparar la presión ejercida en $\mathrm{mmHg}$ y $\mathrm{cmH}_{2} \mathrm{O}$ del neumotaponamiento entre las diferentes categorías del personal que insufló no se observó una diferencia significativa, ANOVA 0.066 y 0.074 respectivamente (Fig. 2).

El volumen en $\mathrm{ml}$ para el neumotaponamiento en el grupo de pacientes normoinsuflados fue de $5 \pm 0.7 \mathrm{ml}$
Tabla 1. Personal que realizó el procedimiento de intubación endotraqueal e insuflación del neumotaponamiento

\begin{tabular}{|l|c|c|c|}
\hline Categoría & Frecuencia & Porcentaje & $\begin{array}{c}\text { Intervalo de } \\
\text { confianza 95\% }\end{array}$ \\
\hline $\begin{array}{l}\text { Médico de } \\
\text { base no } \\
\text { urgenciólogo }\end{array}$ & 3 & 5.0 & $-0.0068-1.068$ \\
\hline $\begin{array}{l}\text { Residente de } \\
\text { primer año }\end{array}$ & 8 & 13.3 & $0.0448-0.2219$ \\
\hline $\begin{array}{l}\text { Residente de } \\
\text { segundo año }\end{array}$ & 6 & 10.0 & $0.0218-0.1782$ \\
\hline $\begin{array}{l}\text { Residente de } \\
\text { tercer año }\end{array}$ & 38 & 63.3 & $0.5078-0.7589$ \\
\hline $\begin{array}{l}\text { Médico } \\
\text { urgenciólogo }\end{array}$ & 5 & 8.3 & $0.0113-0.1553$ \\
\hline
\end{tabular}

Tabla 2. Frecuencia del volumen administrado al neumotaponamiento representado en mililitros

\begin{tabular}{|l|c|c|c|}
\hline $\begin{array}{l}\text { Volumen } \\
\text { en ml }\end{array}$ & Frecuencia & Porcentaje & $\begin{array}{c}\text { Intervalo de confianza } \\
95 \%\end{array}$ \\
\hline 4 & 3 & 5.0 & $-0.0068-0.1068$ \\
\hline 5 & 22 & 36.7 & $0.2411-0.4922$ \\
\hline 6 & 8 & 13.3 & $0.0448-0.2219$ \\
\hline 7 & 16 & 26.7 & $0.1515-0.3819$ \\
\hline 8 & 8 & 13.3 & $0.0448-0.2219$ \\
\hline 10 & 3 & 5.0 & $-0.0068-0.1068$ \\
\hline
\end{tabular}

(IC 95\%: 4.5-5.3), mientras que para el grupo suprainsuflado fue de $6 \pm 1 \mathrm{ml}$ (IC 95\%: 6.3-7.1).

\section{Discusión}

En el servicio de urgencias, los pacientes que requieren apoyo ventilatorio tienen una mayor probabilidad de hipoxemia profunda preintubación, así como de paro cardiorrespiratorio y muerte. Dentro de los principales factores relacionados están el tiempo para reunir al equipo, realizar una evaluación, preoxigenación o estabilización previa?.

La presión de insuflado del neumotaponamiento se determina regularmente de manera subjetiva, por medio de la palpación o digitopresión, al no contar siempre con un manómetro aneroide en el servicio de urgencias. 


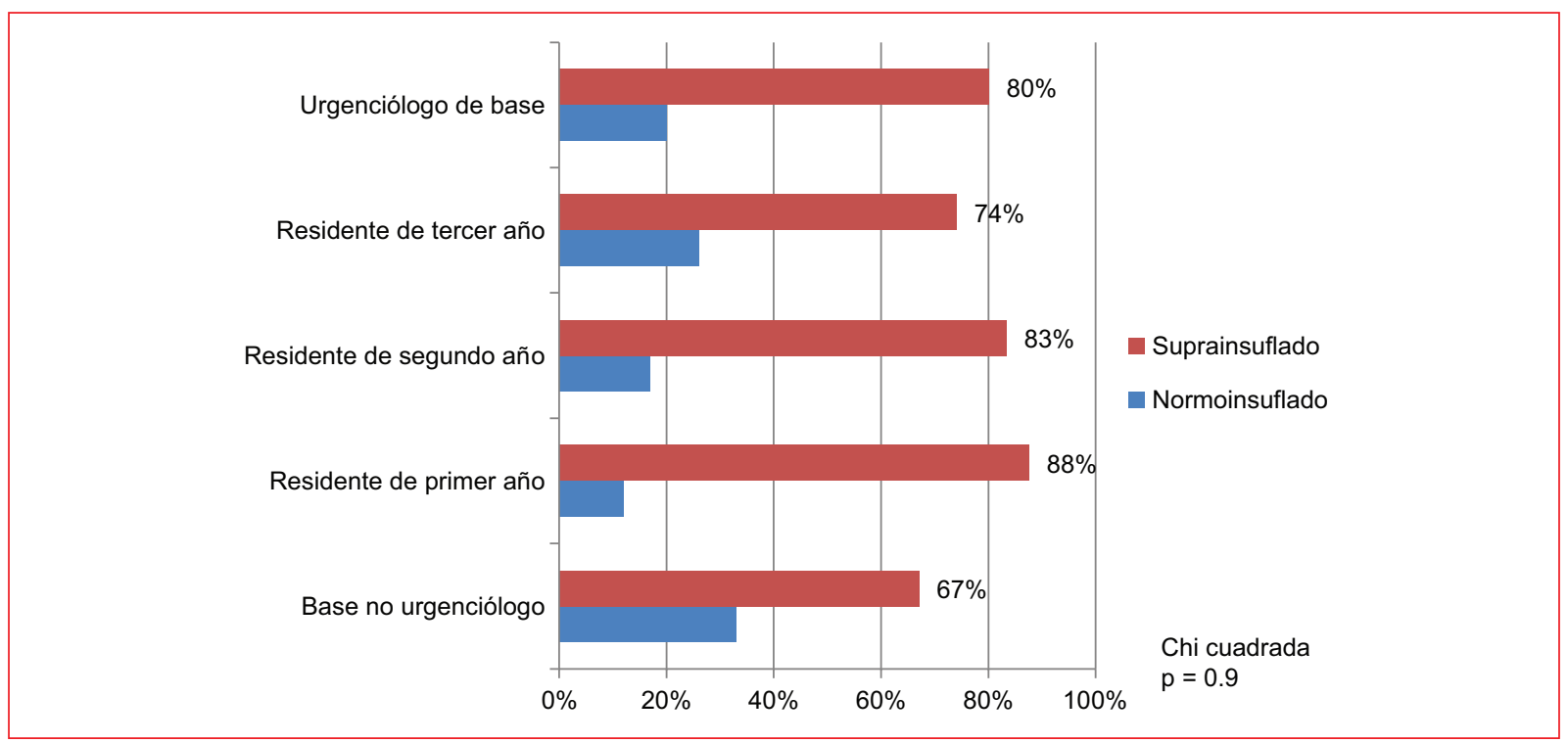

Figura 1. Relación entre insuflado y personal que realiza el procedimiento.

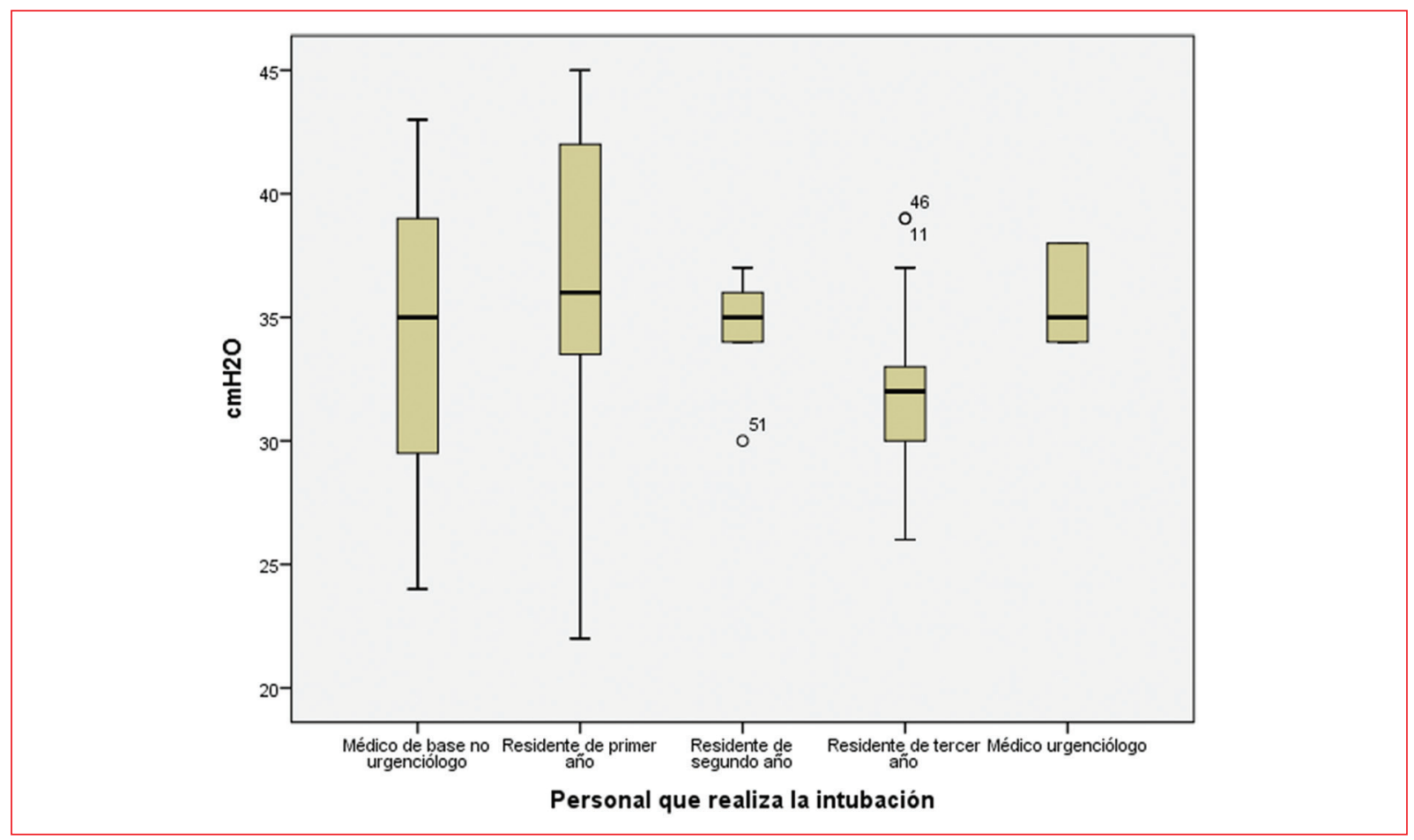

Figura 2. Relación entre la presión de insuflado en $\mathrm{cmH}_{2} \mathrm{O}$ y personal que realiza el procedimiento.

Coincidiendo con todos los estudios revisados, en la mayoría de los pacientes intubados, la presión del manguito del TET se encuentra fuera del rango recomendado, manifestando la necesidad de una medición directa por manometría de las presiones intratraqueales ${ }^{5,6}$.
Se ha reportado que al aumentar la presión en el globo endotraqueal y por ende a la tráquea, se pueden llegar a generar complicaciones de leves a severas, incluyendo afectaciones de la supraglótica, parálisis de cuerdas vocales, estenosis subglótica, microaspiración 
por infrainsuflado (aspiración silenciosa), tos, disfonía, disfagia postextubación, neumonías asociadas a la ventilación, lesiones en la mucosa de la tráquea o incluso ruptura traqueal por suprainsuflación, con la consecuente prolongación de la estancia hospitalaria ${ }^{8-10}$.

Los resultados del estudio no demostraron ninguna infrainsuflación, a diferencia de lo reportado por Vilma, et al. ${ }^{11}$, quienes reportan casi la mitad de los casos con infrainsuflación (presión media de 24 vs. $33 \mathrm{cmmH}_{2} \mathrm{O}$ ) y Félix Ruiz, et al. ${ }^{5}$, quienes mencionan que el $17 \%$ de la población incluida tenía presión subóptima y el $27.7 \%$ con la técnica de fuga mínima. Coincidimos en que solo una cuarta parte lo insufla en rango normal, lo que puede deberse a que consideraron médicos con especialidad en anestesiología, mientras que nosotros incluimos personal de diferentes categorías, aparte de las características de los pacientes (cirugías programadas vs. urgencias) y diferencia en la técnica de medición de la presión de insuflado.

Sengupta, et al. ${ }^{6}$ compararon la presión del manguito del TET en pacientes bajo anestesia general en tres hospitales, reportando un normoinsuflado en el $27 \%$ de los casos, además de mostrar que ni la morfología del paciente, ni la institución, ni la experiencia del proveedor de anestesia o el tamaño del tubo influyeron en la presión media del manguito. Coincidiendo con los resultados obtenidos en que no se encontró diferencia significativa entre las distintas categorías del personal que manipuló el globo, sin embargo a pesar de que estadísticamente no fue significativo, clínicamente la frecuencia de sobreinsuflación fue menor en el grupo de personal de base no urgenciólogo, siendo estos en gran parte anestesiólogos.

Velasco-Sanz, et al. ${ }^{12}$ analizaron el control de la presión del neumotaponamiento en la unidad de cuidados intensivos por parte del personal de enfermería que maneja el control del manguito traqueal; compararon cuatro momentos, antes y después de la capacitación en el uso de manómetro y manejo del neumotaponamiento, demostrando una relación positiva entre la capacitación y las medidas en rangos normales, y sugiriendo el uso del manómetro no solo al momento de intubar, sino también en el mantenimiento para disminuir el índice de complicaciones en la ventilación mecánica asistida.

Sin embargo, con base en los resultados no hay diferencia estadística entre las categorías, lo que sugiere que no solo es la capacitación lo que va a determinar el insuflado inadecuado.

En estudios recientes se ha comparado el uso del insuflado contra no insuflado del neumotaponamiento, encontrando mayor beneficio en aquellos pacientes en los cuales se utilizó insuflación; a pesar de las diferencias anatómicas, se pueden compensar las fugas, aportar presión positiva a la vía aérea, disminuir la tasa de reintubación y broncoaspiración. La desventaja es el riesgo de daño a la mucosa traqueal desde los primeros 15 minutos de iniciado el apoyo mecánico ventilatorio, sobre todo en aquellos pacientes en los cuales no se realizó una medición óptima del insuflado del neumotaponamiento ${ }^{13,14}$.

Min, et al. ${ }^{15}$ determinaron los patrones de cambios de presión del manguito en relación con varias cantidades de inflado, indicando que el inflado con $3 \mathrm{ml}$ de aire produjo una presión entre 20 y $30 \mathrm{cmH}_{2} \mathrm{O}$ para el tubo de 7.5/14 mm, mientras que Sridermma, et al. ${ }^{16}$ sugieren que para alcanzar una presión de $25 \mathrm{cmH}_{2} \mathrm{O}$ se requiere de $7.1 \mathrm{ml}$. En el presente estudio la mayoría utilizó $5 \mathrm{ml}$ de aire (rango 4 y $10 \mathrm{ml}$ ) generando una presión entre 22 y $45 \mathrm{cmH}_{2} \mathrm{O}$, en el grupo con pneumotaponamiento normoinsuflado la media fue de $4.5 \mathrm{ml}$.

Desafortunadamente, el uso del manómetro en nuestro medio no es sistemático y con los resultados obtenidos se apoya la importancia de una medición adecuada y objetiva del globo del TET debido al amplio rango en la presión que se encuentra comparado con pacientes cuyas mediciones se realizan de manera subjetiva, mejorando así la seguridad del paciente.

En el presente estudio el principal sesgo fue el de selección en cuanto a los médicos que realizaron el procedimiento, no se consideraron características tales como especialidad, antigüedad, si labora en otra institución, capacitación relacionada con la intubación y uso del manómetro, que pudieran influir en los resultados obtenidos. Tampoco fueron consideradas las características de los pacientes para determinar una vía aérea difícil y si esta influye en la presión del neumotaponamiento o si presentaron complicaciones posteriores a la intubación.

\section{Agradecimientos}

A todos los médicos y pacientes que aceptaron participar en el proyecto.

\section{Financiamiento}

La presente investigación no ha recibido ninguna beca específica de agencias de los sectores públicos, comercial o sin ánimo de lucro. 


\section{Conflicto de intereses}

Los autores no manifiestan conflicto de intereses.

\section{Responsabilidades éticas}

Protección de personas y animales. Los autores declaran que para esta investigación no se han realizado experimentos en seres humanos ni en animales.

Confidencialidad de los datos. Los autores declaran que en este artículo no aparecen datos de pacientes.

Derecho a la privacidad y consentimiento informado. Los autores declaran que en este artículo no aparecen datos de pacientes.

\section{Bibliografía}

1. Giusti GD, Rogari C, Gili A, Nisi F. Cuff pressure monitoring by manual palpation in intubated patients: How accurate is it? A manikin simulation study. Aust Crit Care. 2017;30:234-8.

2. Kumar CM, Steet E, van Zundert TCRV. Measuring endotracheal tube intracuff pressure: no room for complacency. J Clin Monit Comput. 2021;35(1):3-10.

3. Braz JR, Navarro LH, Takata IH, Nascimento-Júnior P. Endotracheal tube cuff pressure: need for precise measurement. Sao Paulo Med J. 1999;117(6):243-7.

4. Ferenczy ED, Stoner MJ, Spencer SP, Gee SW, Scherzer DJ, Tobias JD. Elevated endotracheal tube cuff pressure in the pediatric emergency department. Int J Pediatr Otorhinolaryngol. 2018;113:289-91.
5. Felix Ruiz R, López-Urbina DM, Carrillo-Torres O. Evaluar la precisión de las técnicas subjetivas de insuflación del globo endotraqueal. Rev Mex Anest. 2014;37(2):71-6.

6. Sengupta P, Sessler DI, Maglinger P, Wells S, Vogt A, Durrani J, et al. Endotracheal tube cuff pressure in three hospitals, and the volume required to produce an appropriate cuff pressure. BMC Anesthesiol. 2004;4(1):8.

7. Higgs A, Mc Grath BA, Goddard C, Rangasami J, Suntharalingam G, Gale R, et al. Guidelines for the management of tracheal intubation in critically ill adults. Br J Anaesth. 2018;120(2):323-52

8. Hockey CA, van Zundert AA, Paratz JD. Does objective measurement of tracheal tube cuff pressures minimise adverse effects and maintain accurate cuff pressures? A systematic review and meta-analysis. Anaesth Intensive Care. 2016;44(5):560-70.

9. Pombo-Nava A, Barrios Medellín I, Ortega van Beusekom JM, Calderón Wengermann O, Becerril RPB. Hallazgos laríngeos posteriores a intubación orotraqueal. An Orl Mex. 2011;56(2):96-100.

10. Russek-Portales B, Blanco-Rodríguez G, Álvarez-Neri H, Teyssier-Morales G, Vázquez-Frías R, López-Virgen E, et al. Hallazgos endoscópicos en pacientes con intubación prolongada: Presentación de una serie de casos. An Med Asoc Med Hosp ABC. 2010;55(3):127-37.

11. Muñoz V, Mojica S, Gómez JM, Soto R. Comparación de la presión del manguito del tubo orotraqueal estimada por palpación frente a la medición tomada con un baumanómetro. Rev Cienc Salud. 2011;9(3): 229-36.

12. Velasco Sanz TR, Delgado de la Fuente MR, Sánchez de la Ventana AB, Merino Martínez MR. El control del neumotaponamiento en cuidados intensivos: influencia de la formación de los profesionales de enfermería. Enferm Intensiva. 2015;26(2):40-5.

13. Bhardwaj N. Pediatric cuffed endotracheal tubes. J Anaesthesiol Clin Pharmacol. 2013;29(1):13-8.

14. Chambers NA, Ramgolam A, Sommerfield D, Zhang G, Ledowski T, Thurm M, et al. Cuffed vs. uncuffed tracheal tubes in children: a randomised controlled trial comparing leak, tidal volume and complications. Anaesthesia. 2018;73(2):160-8.

15. $\mathrm{Fu} Y, \mathrm{Xi} X$. [Analysis on risk factors of endotracheal cuff under inflation in mechanically ventilated patients]. Zhonghua Wei Zhong Bing Ji Jiu Yi Xue. 2014;26(12):870-4.

16. Sridermma $S$, Limtangturakool $S$, Wongsurakiat $P$, Thamlikitkul V. Development of appropriate procedures for inflation of endotracheal tube cuff in intubated patients. J Med Assoc Thai. 2007;90(Suppl 2):74-8. 